\title{
Possibilities of urolithiasis crystallodiagnostics
}

\author{
A.K. Martusevich ${ }^{1-3^{*}}$ and L.M. Kozlova ${ }^{1}$ \\ ${ }^{1}$ Kirov State Medical Academy, Kirov, ${ }^{2}$ Nizhny Novgorod State Agricultural Academy, Nizhny Novgorod, \\ ${ }^{3}$ Privolzhsky Federal Medical Research Centre, Nizhny Novgorod, Russia \\ *Corresponding Author: 603070, Russia, Nizhny Novgorod, Verhnevolzhskaya emb. 18/1; \\ tel.: +7-909-144-91-82; e-mail: cryst-mart@yandex.ru
}

(Received November 19, 2016; Accepted January 30, 2017)

\begin{abstract}
Nowadays, one of the most common groups of diseases in veterinary medicine is the urinary system pathology. Urolithiasis is widespread disease, could be found in many species, including cats, dogs, rabbits, guinea-pigs, turtles etc. Despite the large scale of this pathology in animal world, there are some challenges with diagnostic process and diagnosis's verification. The aim of our study is estimation of diagnostic value of advanced urine tesiocrystalloscopy in urolithiasis (by the example of cats). We studied crystallogenic and initiated properties of 24 healthy cats and 32 animals with urolithiasis. Own and initiated crystallogenesis of the urine specimens was studied. For teziographic test we used sodium chloride solution $(0.45 \%, 0.9 \%, 3 \%$ consequently), hydrochloric acid solution $(0.1 \mathrm{H})$ and sodium hydroxide $(0.1 \mathrm{H})$ as a crystal-forming substances. We used the original criterions to estimate crystalloscopic and tezigraphic facias. As the main parameters structure index (SI), crystallizability (Cr), facia's destruction degree (FDD) and edge belt intensity (EB) were used to describe free crystallogenesis, and main tezigraphic coefficient (Q), belt coefficient (B) and FDD were used for the comparative tezigraphy data. Results showed that Cat's urine in normal conditions has moderate crystallogenic activity, but in urolithiasis it acquires high level of crystallizing, with intermedium value of structure index, and significant destruction of crystal-forming elements. A similar changes of physical-chemical biomedium properties are detected during analysis of tezigraphic microslides of urines of cats with urolithiasis, that was prepared using $0.9 \%$ sodium chloride as basis substance. In conclusion, we fixed that tesiocristalloscopic ,pattern“ of cats' urine in urolithiasis significantly transforms into activation of crystal formation and increasing of biomedium's initiating potential. So, the investigation of free or initialized urine crystallization in urolithiasis has diagnostic value.
\end{abstract}

Keywords: Cat; Urine; Crystallization Urolithiasis; Biocrystallomics Available online at http://www.vetmedmosul.org/ijvs

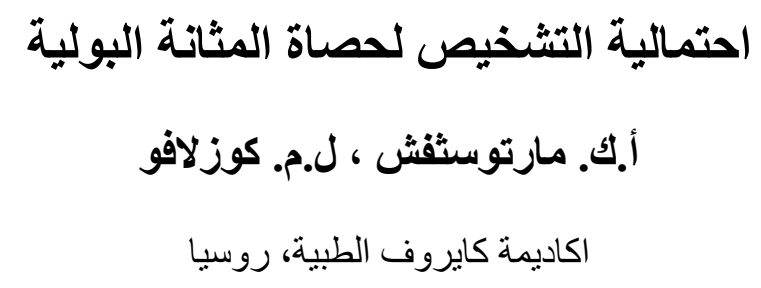

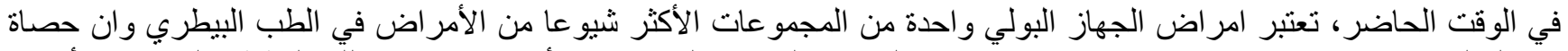

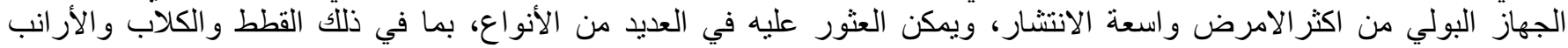

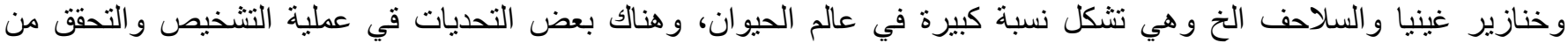

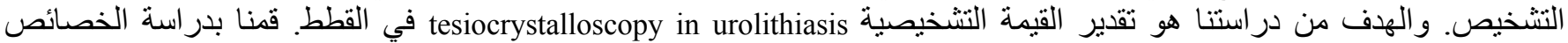

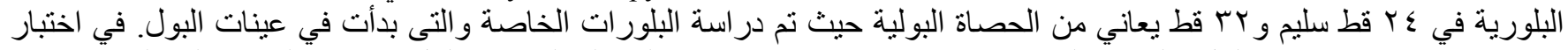

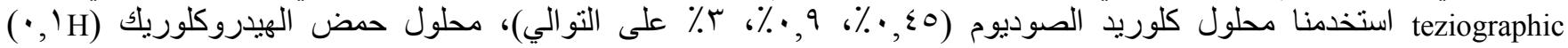




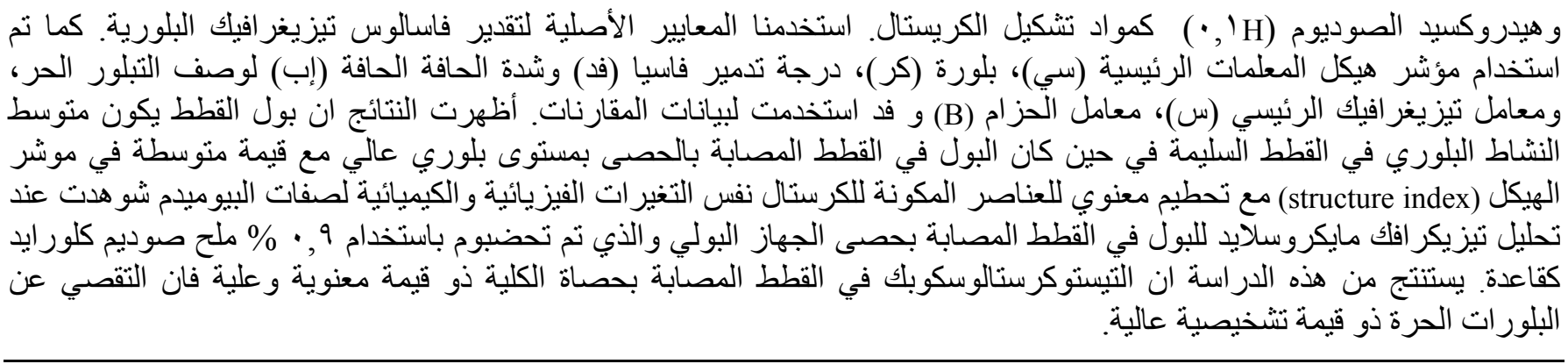

\section{Introduction}

Nowadays, one of the most common groups of diseases in veterinary medicine is the urinary system pathology $(1,2)$. This fact can be confirmed by increasing number of owners of pets with urolithiasis, looking for help in veterinary clinics $(3,4)$. Urolithiasis is widespread disease, could be found in many species, including cats, dogs, rabbits, guinea-pigs, turtles etc (1,4-6). Despite the large scale of this pathology in animal world, there are some challenges with diagnostic process and diagnosis's verification $(1,7)$.

The molecular mechanisms of lithogenesis in urinary system are not completely decoded. There are some theories of urolithes forming (1,7-10): 1. The colloid protection theory, connected with shifts in balance between urinal colloids and crystalloids. 2. The antagonistic ions theory, based on presence in urine ions of magnesium, glucuronic and ascorbic acids, preventing organism from uroliths forming. 3. The organic matrix theory, based on primal forming of protein basis, where, then, urinal crystalloids are deposited.

There are two factors of urolithiasis etiology: a) exogenic (e.g. air temperature, composition of water and soil, and lack of nutrients and vitamins in food); b) endogenic (e.g. hormonal disorders, anatomic anomalies and anomalies of urinary system, digestive system dysfunctions).

Common methods, using in urolithiasis diagnostic process include $(2,3,8,11,12)$ : Clinical evaluation, when some urolithiasis distinctive symptoms (as ischuria, dysuria, pollakiuria, hematuria, hemoglobinuria, leukocyturia, proteinuria) can be revealed. It's important to note, that during clinical evaluation only deep disorders in urinary system can be seen. Also, sometimes this method of animal examining doesn't let to differentiate diagnosis between urolithiasis and other diseases, that can proceed with the same symptoms. Radiography let us to localize urolith. However, there are some significant issues of this method, as impossibility to detect non-contrast uroliths (cystines, urates, phosphates), necessity of preliminary enema and/or sedation, possibility that concrement could be projected on bones or colon, difficulty to detect small concrements and sand. Besides, to make correct diagnosis the high quality of roentgenograms, that determines high quality of radiology equipment, as well as professionalism of physicians are needed. Ultrasonography let us to detect and localize all kinds of uroliths, including small size concrements. This method is safer than radiology. On the other side, low quality of ultrasonography equipment, professional level of physicians in veterinary clinics, high costs and duration of procedure limit widespreading of this method of urolithiasis diagnostics in animals. Ureterocystoscopy - method, based on visual examining distal parts or urinary system, that let to differentiate clinically alike pathological processes, and to detect urethral plugs. Though, practical using of ureterocystoscopy is also limited by high equipment cost that leads to low quality of equipment in veterinary clinic, difficulty and potentialy traumatising of procedure. Laboratory monitoring is one of the easiest to access, fast and cheap method of urolithiasis diagnostics. It includes chemical composition analysis and microscopic estimation of urine sediment. However, to correct laboratory monitoring in veterinary clinics, qualified specialists in laboratory diagnostics are required.

Relying on those methods (excluding clinical evaluation) we can mark out some factors, that make difficult to verificate diagnosis „urolithiasis“ in small animals (cats including). Though, there are low informativity, impossibility to use those methods for early stage urolithiasis diagnosis, when diseases isn't clinically manifested yet. It's important to monitoring the disease course, current and final estimation of treatment effectivity, that bound with missing possibility of pathology course observation. We also have to mark out the lack of diagnostic methods in veterinary medicine, that differ urolithiasis from the diseases with clinically similar symptoms, the lack of cheap, fast and accurate method of urolithiasis diagnostics. Among all urolithiasis diagnostic methods the laboratory urine monitoring seems to be most perspective.

During last years many authors suggested to use analysis of urine sediment in animals, potentially struggled 
with urolithiasis. This method has certain diagnostic value, but also loses information, connected with incomplete deposition of number of organic and non-organic biomedium components, that causes necessity of searching and exploring new diagnostic technologies, that don't associated with biomaterial component's deposition. (1316).

According to this fact, it seems perspective to look at methods, based on biosubstrates dehydrotation, that has already realised with human's urine and described as the collection of biocrystalloscopic exploring methods (16-20). Unfortunately, today these technologies haven't wide application in animals, and there are just a few works in this part of biology and veterinary medicine. Besides, the process of crystal-forming in urolithiasis hasn't been explored before with those methods $(18,21)$.

The aforesaid let us to formulate the purpose of our study as estimation of diagnostic value of advanced urine tesiocrystalloscopy in urolithiasis (by the example of cats).

\section{Materials and methods}

We used urine specimens from 24 healthy cats and 32 cats with clinically significant urolithiasis. Our study was approved with Local Ethic Committee of Nizhny Novgorod State Agricultural Academy (Nizhny Novgorod, Russia).

Own and initiated crystallogenesis of the urine specimens was studied. For teziographic test we used sodium chloride solution $(0.45 \%, 0.9 \%, 3 \%$ consequently), hydrochloric acid solution $(0.1 \mathrm{H})$ and sodium hydroxide $(0.1 \mathrm{H})$ as a crystal-forming substances. We used the original criterions to estimate crystalloscopic and tezigraphic facias $(18,21)$. As the main parameters structure index (SI), crystallizability (Cr), facia's destruction degree (FDD) and edge belt intensity (EB) were used to describe free crystallogenesis, and main tezigraphic coefficient (Q), belt coefficient (B) and FDD were used for the comparative tezigraphy data $(18,21)$. These indices have the same information sense at crystalloscopic and tezigraphic specimens deciphering. All parameters for crystalloscopic facia (SI, Cr and FDD) were estimated in straight point scale with a minimum corresponding to 0 points and a maximum corresponding to 3 points. Calculation of FDD for tezigraphic facias was similar to crystalloscopic one. Main tezigraphic coefficient (Q) was calculated a as the number of crystallization centers in the sample, including biological fluid and $0.9 \%$ sodium chloride solution, and the sample obtained only from sodium chloride. Belt coefficient (B) is the ratio of the maximum and minimum diameters of the belts formed in teziographic facia.

Statistical analysis of the data was performed with Statistica 6.0 program. Data were expressed as means \pm SE, the Student's t-test was used for detection of statistical difference.

\section{Results and discussion}

A specificity of healthy animals' metabolism is urine saturation with organic and non-organic crystal forming substances, able to form concrements in urolithiasis $(1,3,16)$. Those characteristics provide diagnostically meaningful changes of crystallogenic properties and initiating potential of cat's urine, that was expressed in statistically significant shifting of particular biomedium's "tesiocrystalloscopic pattern", causing abrupt crystal formation activation $(\mathrm{P}<0,05$ - for number of estimated visuametric parameters).

Cat's urine in normal conditions has moderate crystallogenic activity, but in urolithiasis it acquires high level of crystallizing, with intermedium value of structure index, and significant destruction of forming crystal elements (fig. 1).

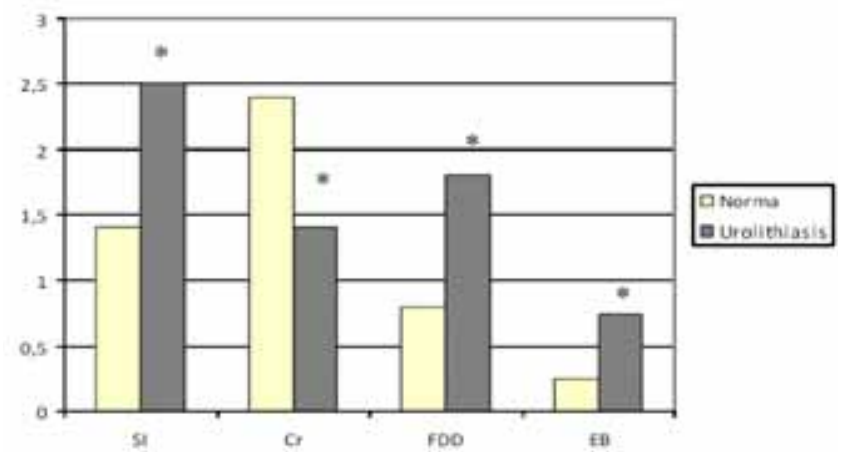

Fig. 1: Results of parametric estimation of urine crystallization of healthy cats and cats with urolithiasis ( $\left\langle^{*}\right\rangle$ - level of statistically significant differences is $\mathrm{P}<0.05$ ).

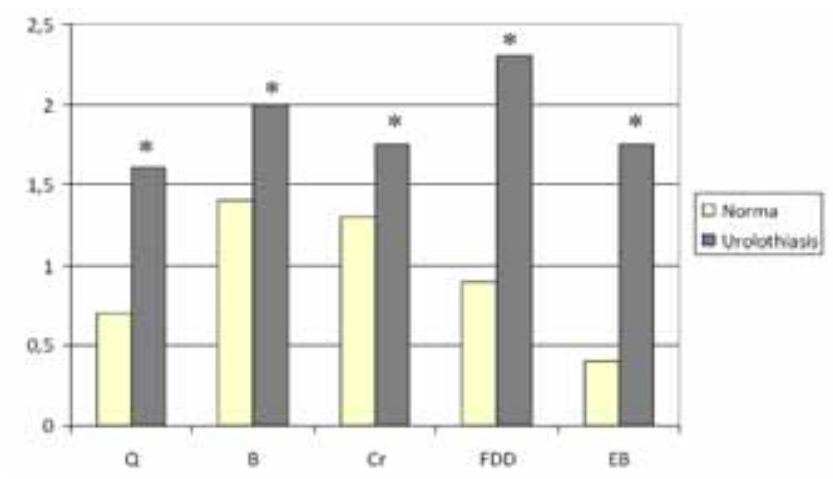

Fig. 2: Results of urine of healthy cats and cats with urolithiasis teziography (basic substance 0,9\% Natrii chloride solution; $\left\langle{ }^{*}\right\rangle-$ level of statistically significant differences $\mathrm{P}<0,05$ ). 
These crystallogenic potential changes of studying biomedium in particular pathology indicates about obtaining increasing of crystallogenesis, i.e. there is significant increasing of crystals growth. This fact is indirectly visualised as significant growth of SI $(P<0,05)$, that interpretates as complication of crystal structure from prevalence of single crystal elements to almost complete saturation of crystalloscopic facia with dendrite structures. These increase of structure's volume in sample leads to decreasing of level of crystal's density in sample to 10-20 per field of view $(\mathrm{P}<0,05)$. Pathological disposition of free crystallogenesis's changes in cat's urine in urolithiasis is also confirmed by meaningful increasing of facia destruction degree comparing with healthy animals $(\mathrm{P}<0,05)$.

Urolithiasis is quite often combined with other renal pathology, which could be presented with proteinuria. Also, we determined moderate proteinuria in animals in study group, that was shown by expansion of edge belt zone of microslide (EB; $\mathrm{P}<0,05)$.

A similar changes of physical-chemical biomedium properties are detected during analysis of teziographic microslides of urines of cats with urolithiasis, that was prepared using $0,9 \%$ sodium chloride as basis substance (fig. 2). In particular, significant increasing of basic tezigraphic coefficient (Q) from moderate inhibiting to quite obtain crystallogenesis activation of basic substance $(\mathrm{P}<0,05)$ tell us about decreasing of crystallogenic urine stability. The "Q" coefficient is basic quantity crystalforming criterion, in proportion of the quantity of crystallization centres. As well as for nature crystallogenesis, in tezigraphic test for urine of cats with urolithiasis there are increased facia structurization with prevailing of dendrite elements in sample (the " $\mathrm{Cr}$ " parameter).

Heterogeneity of studying biomaterial in urolithiasis in combine with increased part of protein in it was detected by the level of belt coefficient $P$ and obtains expansion of edge belt zone. Besides, for microslides of studying group there are significantly higher level of crystals destruction, that also confirmed by level of facia destruction degree (FDD) for cats with urolithiasis $(\mathrm{P}<0,05)$.

Due to make more complete characteristic of initiative urine properties in urolithiasis, it was made the tezigraphic analysis using several basic substances, created different biocrystallogenesis microenvironments (fig. 3). Presence of investigated pathology determines increasing of biosubstrates initiating potential, was revealed as increasing of basic tezigraphic coefficient, and also as significant increasing of destruction level of crystal and amorphic structures in sample.

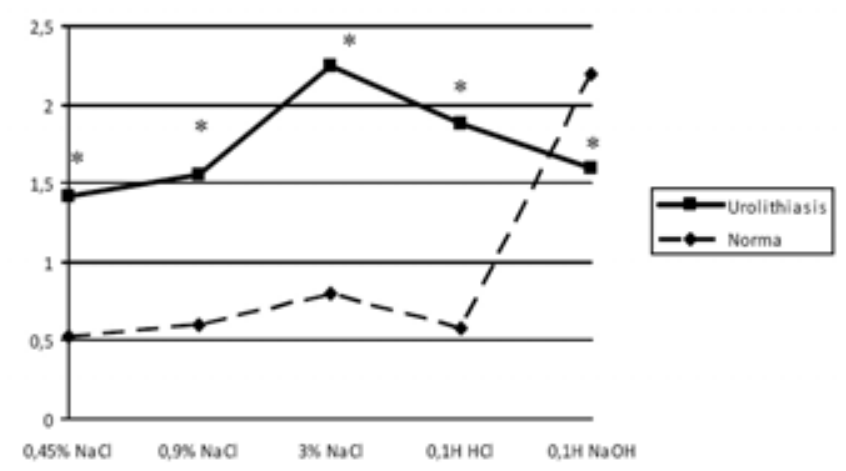

Fig. 3: Initiative profile of cat's urine in studying and control groups $\left(\left\langle^{*}\right)^{*}-\right.$ level of statistically significant differences $\mathrm{P}<0,05)$.

According to figure 3, the initiative potential of urine of cats with urolithiasis is higher than in healthy animals. This is shown by reliably higher value of basic tesigraphic fasia coefficient in studying group comparing to most part of used crystal-forming substances (sodium chloride solutions of any osmolarity; hydrochloric acid solution $0,1 \mathrm{H}$ ), besides parallel crystallisation with basic substance with alkaline media (sodium hydroxide solution $0,1 \mathrm{H}$ ). It could be caused by the fact that urine of major part of examined animals with urolithiasis has as acid $\mathrm{pH}$, and while its neutralization the initiating potential hasn't full expression.

Thus, there was presented principally new method of urolithiasis diagnostics $\quad-\quad$ personalized urocrystallodiagnostics, based on comparing studying dehydrated crystallisation of urine of healthy animals and animals with urinary system pathology. This method could be used as for intact urine as for its components (suprasediment fraction, sediment). It let us to analyse current animal state, to make differential diagnostics of disorders of particular localisation, to monitor its dynamics, to provide primal and secondary prevention, and also to personalize and to track premature and correcting therapy. We suggest to consider all above-mentioned as part of new laboratory diagnostic method - veterinary biocrystallomics.

\section{Conclusion}

Specifity of own and initiated crystallogenesis of healthy and mature cats were approached. Tesiocristalloscopic „pattern“ of cats' urine in urolithiasis significantly transforms into activation of crystal formation and increasing of biomedium's initiating potential. Investigation of free or initialized urine crystallisation in urolithiasis has diagnostic and prognostic value. 


\section{References}

1. Bartges JW, Callens AJ. Urolithiasis. Vet. Clin. North Am. Small Anim Pract., 2015;45(4):747-768.

2. Lund HS, Krontveit RI, Halvorsen I, Eggertsdóttir AV. Evaluation of urinalyses from untreated adult cats with lower urinary tract disease and healthy control cats: predictive abilities and clinical relevance. $\mathrm{J}$ Feline Med Surg. 2013;15(12):1086-1097.

3. Furman E., Hooijberg EH, Leidinger E. et al. Hereditary xanthinuria and urolithiasis in a domestic shorthair cat. Comp Clin Path. 2015;4(6):1325-1329.

4. Nevins JR, Mai W, Thomas E. Association between ultrasound and clinical findings in 87 cats with urethral obstruction. Vet Radiol Ultrasound. 2015;56(4):439-447.

5. Gerber B, Brandenberger-Schenk F, Rothenanger E, Müller C. Uroliths of cats in Switzerland from 2002 to 2009. Schweiz Arch Tierheilkd. 2016;158(10):711-716.

6. Hesse A, Orzekowsky H, Frenk M, Neiger R. Epidemiological data of urinary stones in cats between 1981 and 2008. Tierarztl Prax Ausg K Kleintiere Heimtiere. 2012;40(2):95-101.

7. Albasan H, Osborne CA, Lulich JP, Lekcharoensuk C. Risk factors for urate uroliths in cats. J Am Vet Med Assoc. 2012;240(7):842-847.

8. Dijcker JC, Kummeling A, Hagen-Plantinga EA, Hendriks WH. Urinary oxalate and calcium excretion by dogs and cats diagnosed with calcium oxalate urolithiasis. Vet Rec. 2012;171(25):646.

9. Raditic DM. Complementary and integrative therapies for lower urinary tract diseases. Vet Clin North Am Small Anim Pract. 2015;45(4):857-878.

10. van Houten D. Influence of animal feed on the development of calcium oxalate stones in the urinary tract in cats is different that expected. Tijdschr Diergeneeskd. 2013;138(5):306-307.
11. Blanco F, Ortiz-Alías P, López-Mesas M, Valiente M. High precision mapping of kidney stones using $\mu$-IR spectroscopy to determine urinary lithogenesis. J Biophotonics. 2015;8(6):457-465.

12. Pinel CB, Monnet E., Reems MR. Laparoscopic-assisted cystotomy for urolith removal in dogs and cats - 23 cases. Can Vet J 2013;54(1):36-41.

13. Lulich JP, Osborne CA, Carvalho M, Nakagawa Y. Effects of a urolith prevention diet on urine compositions of glycosaminoglycans, Tamm-Horsfall glycoprotein, and nephrocalcin in cats with calcium oxalate urolithiasis. Am J Vet Res. 2012;73(3):447-451.

14. Takahashi F, Mochizuki M, Yogo T. et al. The silicon concentration in cat urine and its relationship with other elements. J Vet Med Sci. 2014;76(4):569-572.

15. Shabalin VN, Shatokhina SN. Morphology of the human biological fluids. Moscow: Khrisopraz; 2001.p.302.

16. Zaleski MG. Dispersion of mineral and protein components in facia of urine and "Lythos-system" diagnosticum mixture drops. Herald New Med Technol. 2005;12(2):93-94.

17. Denisov AB. Algorithm for evaluation of crystal figures obtained after drying of mixed saliva. Bull Exp Biol Med. 2004;138(1):30-33.

18. Martusevich AK, Kamakin NF. Crystallography of biological fluid as a method for evaluating its physicochemical characteristics. Bull Exp Biol Med. 2007;143(3):385-388.

19. Savina LV. Crystalloscopic structures of the healthy and sick person blood serum. Krasnodar: Sovetsakya Kuban; 1999.p.244.

20. Tarusinov GA. Urine crystallographic study in diagnostics of diffuse connective tissue diseases in children. Pediatrics. 1994;1:55-57.

21. Martusevich AK, Samodelkin AG, Kovaleva LK. Experimental investigation of reversible modulation of biological fluids crystallogenesis. J Biomed Sci Engin. 2016;9(1):1-6. 